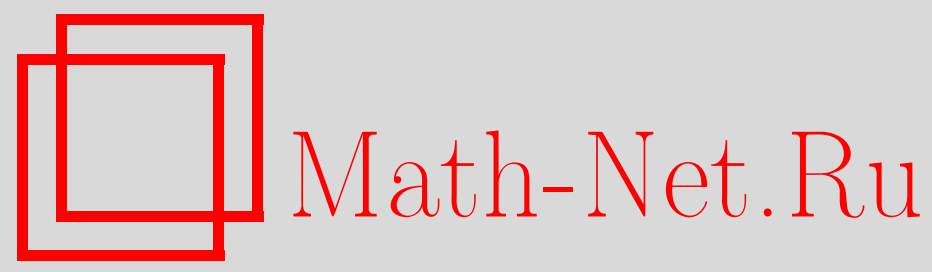

А. А. Туганбаев, Максимальные подмодули и локально совершенные кольца, Матем. заметки, 1998, том 64, выпуск 1, 136-142

DOI: https://doi.org/10.4213/mzm1379

Использование Общероссийского математического портала Math-Net.Ru подразумевает, что вы прочитали и согласны с пользовательским соглашением http://www.mathnet.ru/rus/agreement

Параметры загрузки:

IP: 52.90 .164 .192

26 апреля 2023 г., 12:04:33 


\section{МАКСИМАЛЬНЫЕ ПОДМОДУЛИ \\ И ЛОКАЛЬНО СОВЕРШЕННЫЕ КОЛЬЦА}

\section{А. А. Туганбаев}

Колцо, над которым каждый ненулевой правый (левый) модуль обладает максимальным подмодулем, называется правым (левым) кольцом Басса. Для широкого класса колец, включающего в себя все колца, являющиеся конечнопорожденными модулями над своими центрами, доказана эквивалентность следующих условий:

(1) $A$ - правое кольцо Басса;

(2) $A$ - левое кольцо Басса;

(3) $A / J(A)$ - регулярное кольцо, $J(A)-t$-нильпотентньй справа и слева идеал.

Библиограффия: 10 названий.

Все кольца предполагаются ассоциативными и с ненулевой единицей. Через $C(A)$, $J(A)$ и $\max (A)$ обозначаются соответственно центр, радикал Джекобсона и множество всех максимальных идеалов кольца $A$. Кольца, над которыми каждый ненулевой правый модуль обладает максимальным подмодулем, называются правыми кольиами Басса. В [1], [2] доказан следуюший результат:

Tеорема A [1], [2]. Если $A$ - коммутативное кольцо, то $A-$ кольцо Басса $\Longleftrightarrow$ $A / J(A)-$ регулярное кольцо, $J(A)-$ исчезающий идеал $\Longleftrightarrow A_{M}$ - совершенное кольцо для любого $M \in \max (A) \Longleftrightarrow A_{M}-$ кольцо Басса для любого $M \in \max (A)$.

Основным результатом данной работы является теорема 1.

ТЕОрема 1. Пусть $A$ - локально модульно конечное кольцо с иентром $C$ (әто так, например, если $A$ - конечнопорожсденный $C$-модуль). Тогда равносильны условия:

(1) A - правое кольио Басса;

(2) $A$ - левое кольцо Басса;

(3) $A$ - локально бассово справа кольцо;

(4) $A$ - локально бассово слева кольцо;

(5) $A$ - локально совершенное справа кольцо;

(6) $A$ - локально совершенное слева кольцо;

(7) $C$ - локально совершенное кольио;

(8) $C / J(C)$ - регулярное кольио, $J(C)$ - исчезающий идеал;

(9) $A$ - строго т-регулярно, $J(A)$ - исчезающий слева идеал;

(10) $A$ - строго т-регулярно, $J(A)$ - исчезающий справа идеал. 
Доказательство теоремы 1 разбито на ряд результатов, некоторые из которых представляют самостоятельный интерес. Приведем необходимые определения и обозначения. Слова типа "кольцо Басса" означают, что соответствующие условия выполнены справа и слева. Кольцо $A$ назьвается модульно конечным, если $A$ - конечнопорожденньй модуль над своим центром. Кольцо $A$ назьвается ортогонально конечным, если $A$ не содержит бесконечного множества ненулевых ортогональных идемпотентов. Идеал $B$ кольца $A$ назьвается исчезающим слева (справа), если для любой последовательности $\left\{b_{n}\right\}_{n=1}^{\infty}$ найдется номер $n$ такой, что $b_{n} \cdots b_{1}=0\left(b_{1} \cdots b_{n}=0\right)$. Кольцо $A$ назьвается совершенным справа (слева), если $A$ - полулокальное кольцо и $J(A)$ - исчезающий слева (справа) идеал. Кольцо $A$ назьвается строго $\pi$-регулярным при выполнении следующих эквивалентных [3] условий:

(1) для любого $a \in A$ существует $n$ такое, что $a^{n} \in a^{n+1} A$;

(2) для любого $a \in A$ существует $n$ такое, что $a^{n} \in A a^{n+1}$.

Кольцо $A$ назьвается $\pi$-регулярным, если для любого $a \in A$ найдется $n$ такое, что $a^{n} \in a^{n} A a^{n}$. Любое строго $\pi$-регулярное кольцо $\pi$-регулярно [4]. Кольцо $A$ называется локально совершенным справа (локально ортогонально конечным, локально модульно-конечным локально бассовым справа и т.п.), если для любого $M \in \max (C(A))$ кольцо частных $A_{M}$ является совершенным справа (ортогонально конечным, модульно конечным, правьм кольцом Басса и т.п.). Модульно конечные кольца являются локально модульно-конечньми кольцами. Коммутативное кольцо $A$ назьвается нульмерным при выполнении следующих эквивалентных [5] условий:

(1) все идеалы кольца $A$ простые;

(2) $A / J(A)$ - регулярное кольцо, $J(A)$ - нильидеал.

Лемма 1. Для кольца А равносильны условия:

(1) $А$ - правое кольио Басса;

(2) $A / J(A)$ - правое кольцо Басса, $J(A)$ - исчезающий слева идеал;

(3) все факторкольца кольиа $А$ являются правыми кольцами Басса.

В лемме 1 эквивалентность $(1) \Longleftrightarrow(2)$ доказана в [6, с. 470], а эквивалентность $(1) \Longleftrightarrow(3)$ проверяется непосредственно.

Лемма 2. Для кольца А равносильны условия:

(1) A - строго т-регулярное кольио;

(2) все первичные факторкольца кольца $A$ строго т-регулярны;

(3) факторкольио кольца $A$ по его первичному радикалу является строго т-регулярным кольцом;

(4) все факторкольиа кольиа $A$ по минимальным первичным идеалам строго

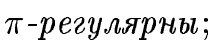

(5) все факторкольца кольиа А строго т-регулярны.

В лемме 2 эквивалентность условий (1) и (2) доказана в [7]. Импликации (1) $\Longrightarrow(3)$, $(3) \Longrightarrow(4),(4) \Longrightarrow(2),(1) \Longrightarrow(5),(5) \Longrightarrow(2)$ следуют из того, что факторкольца строго $\pi$-регулярных колец являются строго $\pi$-регулярными кольцами, причем каждый первичный идеал содержит минимальный первичньй идеал. 
Лемма 3 [6]. $A$ - совершенное справа (слева) кольио $\Longleftrightarrow A-$ полулокальное правое (левое) кольцо Басса.

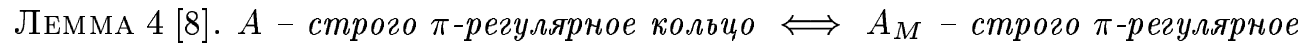
кольио для любого $M \in \max (C(A))$.

ПРЕДЛОЖЕНИЕ 1. Пусть $R$ - унитарное подкольцо центра $C$ кольца А. Равносильны условия:

(1) А - правое кольцо Басса;

(2) для любого мультипликативно замкнутого подмнохества $T \subset C$ кольцо частных $A_{T}$ является правым кольцом Басса;

(3) $A_{M}$ - правое кольцо Басса для любого $M \in \max (R)$, причем в каждом факторкольце кольиа $A$ все иентральные неделители нуля обратимы;

(4) для любого $M \in \max (R)$ верно, что $A_{M}$ - правое кольцо Басса, а канонический гомоморфизм $A \rightarrow A_{M}$ сюрьективен.

ДоКАЗАТЕЛЬСТво. Импликация $(2) \Longrightarrow(1)$ следует из того, что можно положить $T=\{1\}$.

$(1) \Longrightarrow(2)$. Пусть $Q \equiv A_{T}, F$ - ненулевой правый $Q$-модуль, $f: A \rightarrow Q$ - канонический кольцевой гомоморфизм, превращающий $F$ в правьци $A$-модуль. Так как $A$-правое кольцо Басса, $F_{A}$ обладает максимальным подмодулем $N_{A}$. Остается доказать, что $N-$ $Q$-подмодуль. Достаточно для любого $t \in T$ доказать включение $N f(t)^{-1} \subseteq N$. Допустим противное. Так как $f(t) \in C(Q)$, то $N f(t)^{-1}$ - подмодуль в $F_{A}$, не лежащий по предположению в максимальном подмодуле $N_{A}$ модуля $F_{A}$. Поэтому $F=N f(t)^{-1}+N$. Тогда $F f(t)=N+N f(t)=N$. Так как $F-Q$-модуль, имеем $F=F f(t)$. Поэтому $F=N$ и получено противоречие.

$(2) \Longrightarrow(3)$. По лемме 1 достаточно доказать, что $A$ совпадает с кольцом частных $Q$ кольца $A$ относительно множества $T$ всех центральных неделителей нуля. Допустим противное. Тогда ненулевой модуль $(Q / A)_{A}$ обладает максимальным подмодулем $(N / A)_{A}$. Поэтому $N$ - максимальный подмодуль модуля $Q_{A}$. Пусть $h: Q \rightarrow Q / N-$ естественный эпиморфизм. Простой модуль $Q / N$ обладает образующим $h\left(a t^{-1}\right)$, где $a \in A, t \in T$. Поэтому найдется элемент $b \in A$ такой, что $h\left(a t^{-2}\right)=h\left(a t^{-1}\right) b$. Тогда $h\left(a t^{-1}\right)=h\left(a t^{-2}\right) t=h\left(a t^{-1}\right) b t=h(a b) \in h(A) \subseteq h(N)=0$. Поэтому $Q / N=0$ и получено противоречие.

$(3) \Longrightarrow(4)$. Пусть $M \in \max (R), f: A \rightarrow A_{M}$ - канонический гомоморфизм, $h: A \rightarrow$ $A / \operatorname{Ker}(f) \equiv B$ - естественньй эпиморфизм, $t \in R \backslash M$. Тогда $h(t)$ - центральный неделитель нуля в кольце $B$. По условию найдется элемент $a \in A$ такой, что $h(a)=h(t)^{-1}$. Тогда $f(t)^{-1}=f(a) \in f(A)$. Поэтому $A_{T}=f(A)$. Докажем $(4) \Longrightarrow(1)$. Пусть $F_{A}-$ ненулевой $A$-модуль. Тогда найдется такой максимальньй идеал $M$ кольца $R$, что модуль частных $F_{M}$ не равен нулю. По условию ненулевой $A_{M}$-модуль $F_{M}$ обладает максимальным подмодулем $N$. Так как канонический кольцевой гомоморфизм $f: A \rightarrow A_{M}$ по условию сюрьективен, канонический модульньй гомоморфизм $g: F \rightarrow F_{M}$ также сюрьективен. Поэтому достаточно доказать, что $N$ является максимальньп $A$-подмодулем модуля частных $F_{M}$. Пусть $x, y \in F_{M} \backslash N$. Так как $F_{M} / N-$ простой $A_{M}$-модуль, найдется такой элемент $b \in A_{M}=f(A)$, что $x b-y \in N$. Тогда $x f(a)-y \in N$ для некоторого элемента $a \in A$. Поэтому $N$ - максимальньй $A$-подмодуль модуля $F_{M}$. 
СлЕДСТВИЕ 1. Пусть $R$ - унитарное иентральное подкольио т-регулярного кольиа А. Тогда $A-$ правое кольцо Басса $\Longleftrightarrow A_{M}-$ правое кольцо Басса для любого $M \in \max (R)$.

Так как все факторкольца $\pi$-регулярньх колец $\pi$-регулярны, то следствие 1 следует из предложения 1 и того, что в $\pi$-регулярном кольце каждый неделитель нуля обратим.

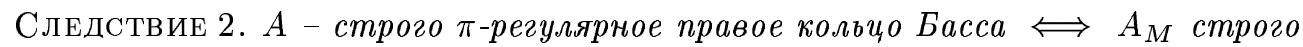
$\pi$-регулярное правое кольцо Басса для любого $M \in \max (C(A))$.

Следствие 2 вытекает из следствия 1 и леммы 4.

Лемма 5. Пусть $R$ - унитарное подкольцо иентра кольца А. Тогда:

(1) $A$ - полупервичное кольцо $\Longleftrightarrow A-$ локально полупервичное кольцо;

(2) $A-$ - регулярное кольцо $\Longleftrightarrow A-$ локально регулярное кольцо;

(3) $A$ - регулярное локально ортогонально конечное кольцо $\Longleftrightarrow A_{M}-$ полупервичное артиново кольио для любого $M \in \max (R)$;

(4) если $S$ - исчезающий идеал кольиа $R$, порождающий в кольце $A$ идеал $B$, то $B$ - исчезающий справа и слева идеал кольца $A$.

ДокАЗАТЕльство. (1) проверяется непосредственно; (2) доказано в [5]; (3) следует из (2) и того, что ортогонально конечное регулярное кольцо является артиновьм. Докажем (4). Верен факт (см. [9, теорема 11.5.5]):

(*) если $X$ - идеал кольца $Y$, то $X$ - исчезающий слева идеал $\Longleftrightarrow N X \neq N$ для каждого ненулевого правого модуля $N_{Y}$.

Пусть $N$ - ненулевой правый $A$-модуль. Из $(*)$ следует, что $N S \neq N$. Но $N S=N B$. Поэтому $N B \neq N$. Из (*) следует, что $B$ - исчезающий слева идеал кольца $A$. Аналогично, $B$ - исчезающий справа идеал.

ЛЕмма 6. Пусть $n$ - натуральное число и кольцо $A$ является $n$-порохсденным модулем над унитарным иентральным подкольиом $R$. Тогда:

(1) для любого $M \in \max (R)$ кольио частных $A_{M}$ является $n$-порожденным модулем над своим локальным иентральным подкольцом $R_{M}$;

(2) $A J(R) \subseteq J(A)$;

(3) $A_{M} J\left(R_{M}\right) \subseteq J\left(A_{M}\right)$ для любого $M \in \max (R)$;

(4) $A_{M}$ - модульно конечное полулокальное $и$, в частности, ортогонально конечное кольио для любого $M \in \max (R)$;

(5) $A$ - регулярное кольцо $\Longleftrightarrow A_{M}$ - полупервичное артиново кольио для любого $M \in \max (R)$

(6) если $R$ - нульмерное кольцо, то $A / J(A)$ - регулярное кольцо, $J(A)$ - нильидеал, $J(R)=R \cap J(A) ;$

(7) если $R$ - регулярное кольио, то для любого $M \in \max (R)$ кольцо $R_{M}$ является полем, а кольцо $A_{M}$ является конечномерной $R_{M}$-алгеброй, размерность которой не превосходит $n$;

(8) если $R$ - регулярное кольцо, то $A / J(A)$ - регулярное кольцо, $J(A)$ - нильпотентный идеал;

(9) если $R$ - полулокальное кольцо, то $A / J(R) A$ - вполне приводимое кольцо, $A$ - полулокальное $u$, в частности, ортогонально конечное кольцо; 
(10) если $R$ - совершенное кольио, то $A$ - совершенное кольцо.

ДокАЗАТЕЛЬСтво. (1) проверяется непосредственно; (2) доказано в [10, глава 3 , следствие 2.5]; (3) вытекает из (2), примененного к $A_{M}$ и $R_{M} ;$ (4) следует из (1), (3) и того, что $\left(A_{M}\right) /\left(A_{M} J\left(R_{M}\right)\right)$ - конечномерная алгебра над полем $R_{M} / J\left(R_{M}\right) ;(5)$ следует из (4) и из леммы $5 ;(6)$ доказано в [5]; (7) следует из $(1)$ и того, что все локализации коммутативного регулярного кольца по максимальным идеалам являются полями; (8) следует из (6) и (7); (9) проверяется с помощью $(2) ;(10)$ следует из (9) и из леммы $5(4)$.

Лемма 7. Совершенное справа полупервичное кольцо $А$ является артиновым кольцом.

Так как кольцо $A$ ортогонально конечно, то лемма 7 вытекает из следующих хорошо известных утверждений: каждьй ненулевой левый идеал совершенного справа кольца содержит минимальный правьй идеал; каждый минимальньй правьй идеал полупервичного кольца порож дается идемпотентом.

ПРЕДЛОЖЕНИЕ 2. ДЛя кольиа А равносильны условия:

(1) $A$ - локально полулокальное правое кольцо Басса;

(2) $A$ - локально полулокальное локально бассово справа кольцо;

(3) $A$ - локально совершенное справа кольцо;

(4) $A$ - локально полулокальное строго т-регулярное правое кольцо Басса.

ДокАЗАТЕЛЬСТво. Импликация (1) $\Longrightarrow(2)$ следует из предложения 1. Импликация $(2) \Longrightarrow(3)$ следует из леммы 3. Импликация $(3) \Longrightarrow(1)$ вытекает из следствия 2 и того, что по леммам 2 и 3 каждое совершенное справа кольцо является строго $\pi$-регулярным правым кольцом Басса. Импликация (4) $\Longrightarrow(1)$ очевидна. Импликация $(1),(3) \Longrightarrow(4)$ следует из леммы 4 и того, что по лемме 2 совершенные справа кольца являются строго $\pi$-регулярными.

СледствИЕ 3. Пусть $A$ - локально модульно конечное кольцо. Тогда $A-n p a-$

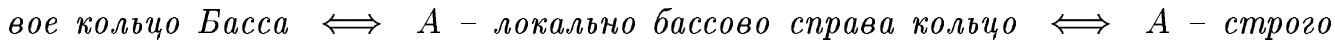

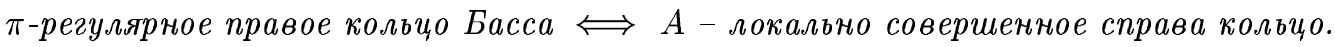

Следствие 3 вытекает из предложения 2 и леммы 6 (4).

СлЕДСТВИЕ 4. Пусть $A$ - локально модульно конечное кольчо. Равносильны условия:

(1) A - полупервичное правое кольио Басса;

(2) $A$ - локально полупервичное локально совершенное справа кольцо;

(3) $A$ - локально полупервичное локально артиново кольцо;

(4) $A$ - регулярное кольцо.

В следствии 4 эквивалентность условий (1) и (2) вытекает из следствия 3 и леммы 4 , а эквивалентность условий (3) и (4) - из лемм 4(3) и 5(5). Импликация (3) $\Longrightarrow(2)$ очевидна. Импликация $(2) \Longrightarrow(3)$ следует из леммы 6 .

СледСтвИЕ 5. Пусть факторкольцо $A / J(A)$ кольца $A$ является локально модульно-конечным кольцом. Тогда $A-$ правое кольцо Басса $\Longleftrightarrow A / J(A)-$ регулярное кольио, $J(A)$ - исчезающий слева идеал.

Следствие 5 вытекает из леммы 1 и следствия 4. 
Лемма 8. Пусть $C$ - иентр строго т-регулярного кольиа А. Тогда $C$-нульмерное кольцо.

ДокАЗАТЕЛЬСТво. $C$ - коммутативное строго $\pi$-регулярное кольцо [4]. Если $P-$ простой идеал кольца $A$, то $A / P$-строго $\pi$-регулярная область. Поэтому $C / P$ - поле, $C$ - нульмерное кольцо.

ПРЕДЛОЖЕНИЕ 3. Пусть кольцо $А$ является конечнопорожденным модулем над своим иентром $C$. Тогда равносильны условия:

(1) $A$ - правое кольио Басса;

(2) $A$ - левое кольчо Басса;

(3) $A$ - локально бассово справа кольцо;

(4) $A$ - локально бассово слева кольцо;

(5) $A$ - локально совершенное справа кольцо;

(6) $A$ - локально совершенное слева кольцо;

(7) $A$ - строго т-регулярное правое кольцо Басса;

(8) $A$ - строго т-регулярное левое кольцо Басса;

(9) $C$ - локально совершенное кольцо;

(10) $C-$ кольио Басса;

(11) $C / J(C)$ - регулярное кольчо, $J(C)$ - исчезающий идеал;

(12) $A / J(A)$ - регулярное кольио, $J(A)$ - исчезающий справа и слева идеал.

ДоказАтЕльство. Так как условие (12) симметрично, достаточно доказать эквивалентность условий $(1),(3),(5),(7),(9),(10),(11)$ и (12). Эквивалентность условий $(1),(3)$ и (5) вытекает из следствия 3 . Так как $A / J(A)$ - модульно конечное кольцо, эквивалентность условий (1) и (12) вытекает из следствия 5. Эквивалентность условий (5) и (7) вытекает из следствия 3. Эквивалентность условий (9), (10), (11) следует из теоремы А.

$(7) \Longrightarrow(11)$. По лемме $8 C$ - нульмерное кольцо. Так как по лемме $6(6) J(C)=$ $C \cap J(A)$, причем по лемме $1 J(A)$ - исчезающий слева идеал, $J(C)$ - исчезающий идеал.

$(11) \Longrightarrow(12)$. По лемме 6(6) $A / J(A)$ - регулярное кольцо. Пусть $h: A \rightarrow A / J(C) A-$ естественный эпиморфизм. Так как кольцо $h(A)$ является конечнопорожденным модулем над коммутативным регулярным кольцом $C / J(C)$, по лемме $6(8) h(J(A))$ - нильпотентный идеал. Кроме того, поскольку $J(C)$ - исчезающий идеал, по лемме $5(4)$ $J(C) A$ - исчезающий справа и слева идеал кольца $A$. Поэтому $J(A)$ - исчезающий справа и слева идеал кольца $A$.

СлЕДСТВИЕ 6. Пусть кольцо А является конечнопорохсденным модулем над своим иентром С. Тогда равносильны условия:

(1) $A$ - совершенное справа кольио;

(2) $A$ - совершенное слева кольцо;

(3) $C$ - совериенное кольио.

Следствие 6 вытекает из предложения 3 , лемм 3 и $6(10)$.

ДокАЗАТЕЛЬСТво ТЕОРЕмЫ 1. Так как условие (8) симметрично, то достаточно доказать эквивалентность условий (1), (3), (5), (7), (8) и (9). Эквивалентность условий 
$(1),(3)$ и (5) доказана в следствии 3 . Эквивалентность условий (7) и (8) следует из теоремы А. Импликация (7) $\Longrightarrow(5)$ вытекает из леммы 6 (10). Импликация (1) $\Longrightarrow(9)$ вытекает из леммы 1 и следствия 3 . Докажем импликацию $(9) \Longrightarrow(8)$. Так как $A-$ локально модульно конечное кольцо, то $J(C) \subseteq J(A)[5]$. Поэтому $J(C)$ - исчезающий идеал. По лемме $8 C / J(C)$ - регулярное кольцо.

\section{СПИСОК ЦИТИРОВАННОЙ ЛИТЕРАТУРЫ}

[1] Hamsher R. M. Commutative rings over which every module has a maximal submodule // Proc. Amer. Math. Soc. 1967. V. 18. №6. P. 1133-1137.

[2] Faith C. Locally perfect commutative rings are those whose modules have maximal submodules // Comm. Algebra. 1995. V. 22. № 13. P. 4885-4886.

[3] Dischinger F. Sur les anneaux fortement $\pi$-reguliers // C. R. Acad. Sci. Paris. Sér. A. 1976. V. 283. P. 571-573.

[4] Azumaya G. Strongly $\pi$-regular rings // J. Fac. Sci. Hokkaido Univ. Ser. I. 1954. V. 13. P. 34-39.

[5] Goodearl K.R., Warfield R. B. Algebras over zero-dimensional rings // Math. Ann. 1976. V. 223. P. $157-168$.

[6] Bass H. Finistic dimension and a homological generalization of semiprimary rings // Trans. Amer. Math. Soc. 1960. V. 95. №3. P. 466-488.

[7] Fisher J.V., Snider R.L. On the Von Neumann regularity of rings with regular prime factor rings // Pacific J. Math. 1974. V. 54. № 1. P. 135-144.

[8] Armendariz E. P., Fisher J. V., Steinberg S. A. Central localizations of regular rings // Proc. Amer. Math. Soc. 1974. V. 46. № 3. P. 315-321.

[9] Каш Ф. Модули и кольца. М.: Мир, 1981.

[10] Басс Х. Алгебраическая $K$-теория. М.: Мир, 1973. 